\title{
BEAM LOADING COMPENSATION USING REAL TIME BUNCH CHARGE INFORMATION FROM A TOROID MONITOR AT FLASH
}

\author{
E. Vogel, Ch. Gerth, W. Koprek, F. Loehl, D. Noelle, H. Schlarb, T. Traber \\ Deutsches Elektronen Synchrotron (DESY), Notkestraße 85, 22607 Hamburg, Germany
}

\section{Abstract}

At pulsed linear accelerators, fast proportional rf control compensates beam loading sufficiently for single or a few bunches. In the case of long bunch trains, additional measures have to be taken commonly by adding a compensation signal to the rf drive signals calculated from the predicted beam intensity.

In contrast to predictive methods, techniques based on real time beam measurements are sensitive to fast changes of the beam intensity and bunch patterns. At FLASH we apply a beam loading compensation scheme based on toroid monitor signals. This paper presents the compensation scheme, the calibration procedure and the effect on the beam.

\section{INTRODUCTION}

The free electron laser in $\underline{\text { Hamburg }}$ (FLASH) accelerates electron bunches to energies of $700 \mathrm{MeV}$ every $200 \mathrm{~ms}$ in batches occupying $800 \mu$ s at most with bunch spacings of $1 \mu$ s or multiple thereof (Fig. 1). While passing an undulator the bunches emit high brilliance coherent light in the vacuum ultraviolet range from 60 to $13 \mathrm{~nm}$. This process is based on the self-amplified spontaneous emission (SASE) effect. Major prerequisites are high electron peak currents of 1 to $2 \mathrm{kA}$ and transverse emittances in the range of 1 to $2 \mu \mathrm{m}$. Starting from $1 \mathrm{nC}$ electron bunches $\left(0.62 \cdot 10^{10}\right.$ particles), the high peak currents are obtained by compressing the bunch length down to the 100 fs range. The compression is done within transverse magnetic chicanes, called bunch compressors.

The bunch compression and the SASE process are sensitive to beam energy variations. Bunch compression requires bunches accelerated off the rf field crest to obtain an energy chirp from the bunch head to the tail. Variations of the rf amplitude and phase in the accelerating modules

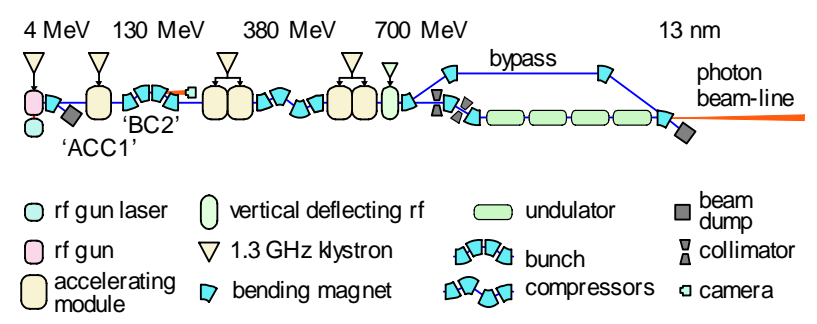

Figure 1: Sketch of the free electron laser in Hamburg (FLASH) facility.

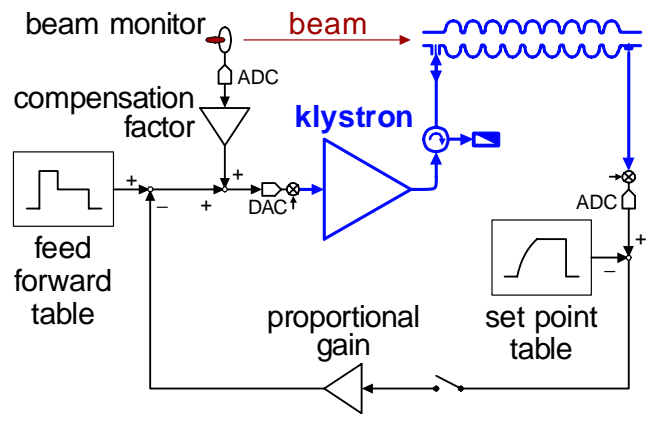

Figure 2: Sketch of the digital control operating the first superconducting rf module (ACC1) at FLASH. DACs in front of the vector modulator $(\otimes)$ and ADCs after down converters $(\otimes)$ are part of the controller (black). The beam loading compensation is calculated from a sampled beam (toroid) monitor signal.

before the bunch compressors change the beam energy and affect the performance of FLASH. Hence, compensating the rf field degradation caused by the beam loading is one major prerequisite to obtain long bunch trains with high peak current creating long series of laser flashes provided to the experiments.

\section{RF CONTROL PRINCIPLE}

Figure 2 shows a sketch of the digital rf control based on the controller board SimCon 3.1 [1]. It regulates $\mathrm{rf}$ amplitude and phase, and sum of the angular pointer components (I and Q) respectively [2], within the first accelerating module (ACC1) at FLASH driven by a pulsed klystron. For simplification only one superconducting nine cell cavity is shown instead of eight and most additional technical components like the klystron pre-amplifier are omitted.

For the optimal transfer of rf power to beam power superconducting cavities are operated with strong coupling $\beta \gg 1$ reducing the unloaded quality factors of $Q_{0} \approx 10^{10}$ to [3]

$$
Q_{\mathrm{L}} \approx \frac{V_{\mathrm{c}}}{I_{\mathrm{dc}} \frac{R}{Q_{0}}} .
$$

The nominal beam current $I_{\mathrm{dc}}=1 \mathrm{nC} \cdot 9 \mathrm{MHz}$, the accelerating voltage $V_{\mathrm{c}}=25 \mathrm{MV}$ and the normalized shunt impedance $R / Q_{0}=1040 \Omega$ of the TESLA type cavities yield $Q_{\mathrm{L}} \approx 3 \cdot 10^{6}$. Due to the high quality factor, the rf drive voltage supplied by the klystron results only in a slow voltage increase inside the cavities while filling. When reach- 
ing the desired voltage $V_{\mathrm{c}}$, beam starts passing the cavity. The voltage reduction due to beam loading

$$
V_{\mathrm{b}} \approx-I_{\mathrm{dc}} Q_{\mathrm{L}} \frac{R}{Q_{0}}
$$

is exactly compensated by the rf drive voltage resulting in a constant voltage level applied to the beam (flat top). In the case of beam current below nominal the drive voltage and the value of the 'feed forward table' have to be reduced at flat top to keep the cavity voltage constant. Calculating the error signal used by the fast proportional control, the slow filling is taken into account using an appropriate 'set point table' (Fig. 2). At the end of flat top the rf drive and the control loop are switched off.

The feed forward table applied at ACC1 reduces the rf drive voltage at flat top to a level appropriate for the situation without beam. When beam is accelerated the beam loading voltage reduction has to be compensated by a correction signal. For single or a few bunches the correction by the fast proportional $\mathrm{rf}$ control alone is sufficient. In the case of long bunch trains additional measures have to be taken commonly by adding a compensation signal to the rf drive signals calculated from the predicted beam intensity. Measuring the beam intensity in real time and applying a compensation signal derived from it takes beam intensity variations automatically into account. The controller at ACC1 offers both methods, here we will discuss only the second one.

\section{BEAM LOADING COMPENSATION}

The toroid monitor used at FLASH provides an analog signal with a duration of about $50 \mathrm{~ns}$ whose amplitude is proportional to the bunch charge. Sampling this signal at $54 \mathrm{MHz}$ results in five samples per bunch. Performing an offset correction based on values sampled before and after the bunch signal and summing the five samples yields a bunch charge proportional value. This value is multiplied by the 'compensation factor' consisting of two angular pointer components I and Q and added to the rf drive signal (Fig. 2).

At FLASH the electron bunches are much shorter than the rf wavelength. Consequently the voltage induced in the cavities depends only linearly on the bunch charge and the cavity parameters. The absolute value of the compensation factor is a function of the loaded quality factor and the normalized shunt impedance (1) and remains constant at standard operation.

When the bunch passage is phase shifted to the cavity rf field both the rf amplitude and phase are affected by the beam loading voltage. The effect on the rf phase has to be compensated by the phasing of the compensation factor.

In practice, the compensation factor can be determined using the following beam based calibration procedure:

The rf field is measured without beam and subsequently with beam and beam loading compensation switched on to

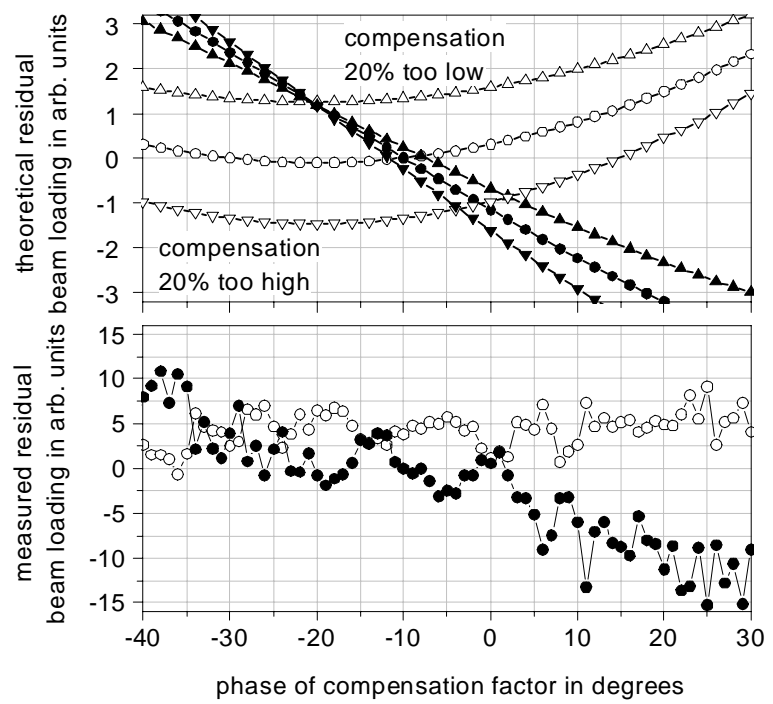

Figure 3: Theoretical and measured residual beam loading voltage (I and Q, plotted black and white) while scanning the phase of the compensation factor and proportional control switched off. With the optimal amplitude for the compensation factor the I and Q curves cross each other at zero amplitude indicating the phase between beam and rf.

determine the rf field difference of these cases. For an optimal compensation factor the rf field difference vanishes. The optimal compensation factor can be found visually by scanning its amplitude and phase while plotting I and Q of the field difference (Fig. 3).

Switching off the proportional rf control while determining the compensation factor results in larger rf field difference values while scanning. This is necessary for calibrating with low intensity beams.

The experimental data taken with proportional rf control off and 30 bunches with $0.7 \mathrm{nC}$ each (Fig. 3) show quite big fluctuations, most probably caused by fluctuations of the klystron output power from rf pulse to pulse. For bunch compression ACC1 accelerates bunches 10 degrees off crest. Hence, both lines should cross at -10 degrees.

\section{RF FIELD STABILITY MEASUREMENT}

The beam energy of $130 \mathrm{MeV}$ at the first bunch compressor (BC2) depends almost exclusively on the rf field amplitude seen by the beam in the first accelerating module (ACC1) due to the comparatively small energy gain of $4 \mathrm{MeV}$ obtained from the rf gun (Fig. 1). Hence, the beam energy stability at $\mathrm{BC} 2$ is a direct measure of the rf amplitude stability in ACC1.

A camera is used to record the spot position fluctuations of the synchrotron radiation emitted by bunches deflected by the third dipole magnet in $\mathrm{BC} 2$ [4]. An automated spot analysis provides the bunch energy and accelerating volt- 


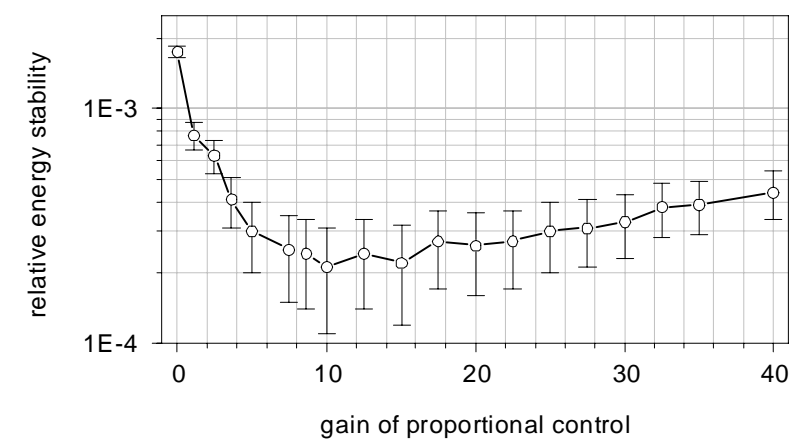

Figure 4: Curve obtained by scanning the gain value of the proportional control while measuring the energy stability of the first bunch. The best energy stability is obtained for gain values between 10 and 15 .

age stability in the bunch compressor BC2 [5]:

$$
\frac{\Delta V_{\mathrm{c}}}{V_{\mathrm{c}}} \approx \frac{\Delta E}{E}=\frac{\left\langle\Delta x_{\mathrm{spot}}\right\rangle}{340 \mathrm{~mm}}
$$

The camera pixel to pixel distance of $80 \mu \mathrm{m}$ results in combination with signal interpolation in a relative measurement resolution of $10^{-4}$.

\section{RESULTS}

The first bunch of a train does not suffer from beam loading effects and its stability is determined by the proportional control only. Scanning the gain value while measuring the energy stability of the first bunch yields the optimal gain for the proportional control (Fig. 4). Starting

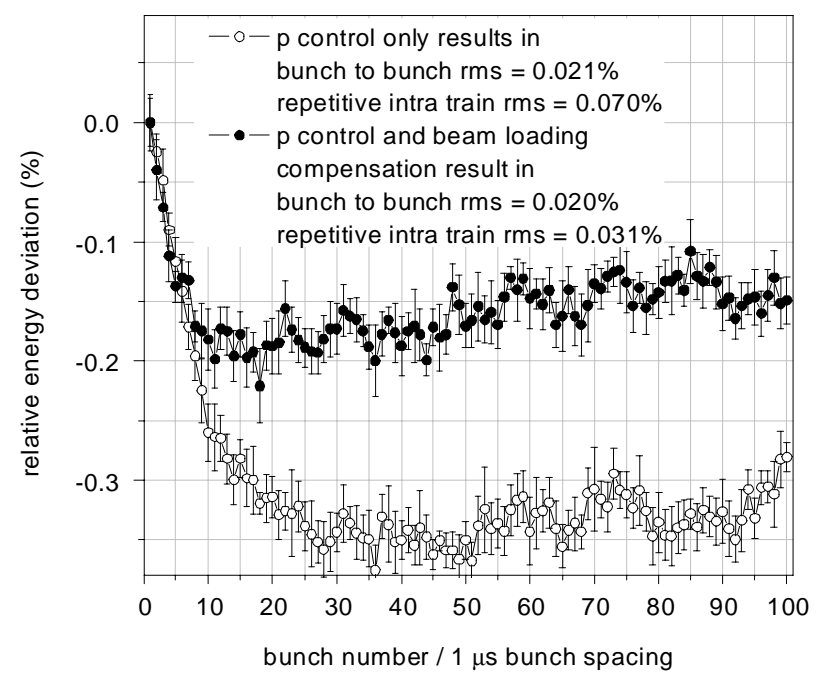

Figure 5: Beam energy determined at $\mathrm{BC} 2$ by recording the transverse position of the synchrotron light spot from bunch to bunch. The 'error bars' indicate the rms values of the individual bunch energies. from zero and increasing the gain value the energy stability increases due to the error suppression by the proportional control. Gain values around 15 result in an energy stability of $\Delta E / E \approx 2 \cdot 10^{-4}$. For higher gain values the stability becomes worse because sensor signal noise is increasingly amplified by the controller and added to the rf drive signal.

Figure 5 shows the relative energy deviation and stability of 100 bunches spaced by $1 \mu$ s using a proportional control gain of 15 and a beam loading calibration factor obtained by the method described. Without beam loading compensation the bunch energy drops by $0.35 \%$ due to the rf voltage drop until the proportional $\mathrm{rf}$ control starts compensating the beam loading. Applying the beam based beam loading compensation the time and the amount of the energy drop are both reduced by a factor of about two. All bunches show a similar relative energy stability and no degradation towards the end of the bunch train.

\section{SUMMARY AND OUTLOOK}

At FLASH beam loading is reliably compensated using real time information obtained from a beam charge monitor. The individual bunch energy is stabilized by the proportional $\mathrm{rf}$ control and the beam loading compensation to a level of $\Delta E / E \approx 2 \cdot 10^{-4}$. Using the beam loading compensation scheme reduces the bunch to bunch energy variation to one half of that obtained with proportional control only. A further improvement may come from a better klystron output regulation while making calibration measurements.

\section{ACKNOWLEDGEMENTS}

We are grateful to Katja Honkavaara, Helen Edwards, Siegfried Schreiber and Bart Faatz for their continuos interest and support of this work. Furthermore, we would like to thank John Maidment for suggestions and careful proofreading.

\section{REFERENCES}

[1] W. Giergusiewicz, W. Jalmuzna, K. Pozniak, N. Ignashin, M. Grecki D.V.M., D. Makowski, T. Jezynski, K. Perkuszewski, K. Czuba, S. Simrock, R. Romaniuk, Proc. of SPIE Vol. 5948, 2005

[2] T. Schilcher, Ph.D. Thesis, University of Hamburg, TESLA Report No. TESLA 1998-20, 1998

[3] H. Padamsee, J. Knobloch, T. Hays, RF Superconductivity for Accelerators (John Wiley \& Sons Inc, 1998)

[4] Ch. Gerth, 'Synchrotron Radiation Monitor for Energy Spectrum Measurements in the Bunch Compressors at FLASH', Proc. of Dipac2007, Venice, May 2007

[5] H. Schlarb, Ch. Gerth, W. Koprek, F. Loehl, E. Vogel, 'Beam Based Measurements of RF Phase and Amplitude Stability at FLASH', Proc. of Dipac2007, Venice, May 2007 